\title{
A 64B/67B Channel Code Implementation and Its Application in Seismic Data Acquisition System
}

\author{
Cheng MA, Shikai WANG, Xue YANG, Jianping XIONG \\ State Key Laboratory of Precision Measurement Technology and Instruments, Department of Precision \\ Instruments, Tsinghua University, Beijing, China
}

\begin{abstract}
Based on guided scrambling technique, the $64 \mathrm{~B} / 67 \mathrm{~B}$ channel code has two remarkable advantages of high bit rate of 64/67 and DC-balance. In this paper, we realize the FPGA implementation of $64 \mathrm{~B} / 67 \mathrm{~B}$ coding scheme and make out a comparison with $8 \mathrm{~B} / 10 \mathrm{~B}$ coding scheme. Testing results indicate that taking resource occupation, bit error rate and effective transmission speed into consideration 64B/67B channel code is the best choice for seismic data acquisition system.
\end{abstract}

KEYWORD: 64B/67B; Guided scrambling; DC-balance; Bit rate

\section{INTRODUCTION}

\subsection{Seismic data acquisition system}

Large-scale Seismic Data Acquisition and Recording (LSDAR) system uses long distance UTP channel for its data transmission. To ensure the reliability and stability in data transmission process, small low frequency content, high efficiency and low error multiplication are some of the typical requirements that the coding scheme needs to fulfill. Fortunately, the guided scrambling (GS) theory proposed a designed method to select best GS coding parameters which can meet the low-frequency requirements of the long distance UTP channel. [1]

\subsection{B/67B channel code}

According to GS theory, if we set the width of source data as 64 bits, choose $d(x)=x^{3}+1$ for scrambling polynomial (number of augmenting bits is 3 ), we will get $64 \mathrm{~B} / 67 \mathrm{~B}$ coding scheme. [2-3] Its bit rate is as high as 0.9552 and also it has an excellent performance of DC-balance characteristics theoretically by using absolute RDS (ABSRDS) as its selection criteria. [4]

The process of $64 \mathrm{~B} / 67 \mathrm{~B}$ coding scheme can be described as 3 steps. Firstly, expanding the data to 67 bits by inserting 3 augmenting bits before the 64 bits of source data; secondly, multiplying the scrambling polynomial with the data generated in step 1 to get candidate codes; finally, picking out the optimal code for outputting from all the candidate codes according to the selection criteria.
Figure 1 shows the 3 steps of $64 \mathrm{~B} / 67 \mathrm{~B}$ coding process. Here, $s(x)$ means 64 bits' source data; $d(x)$ is the scrambling polynomial; $b(x)$ represents for candidate codes.

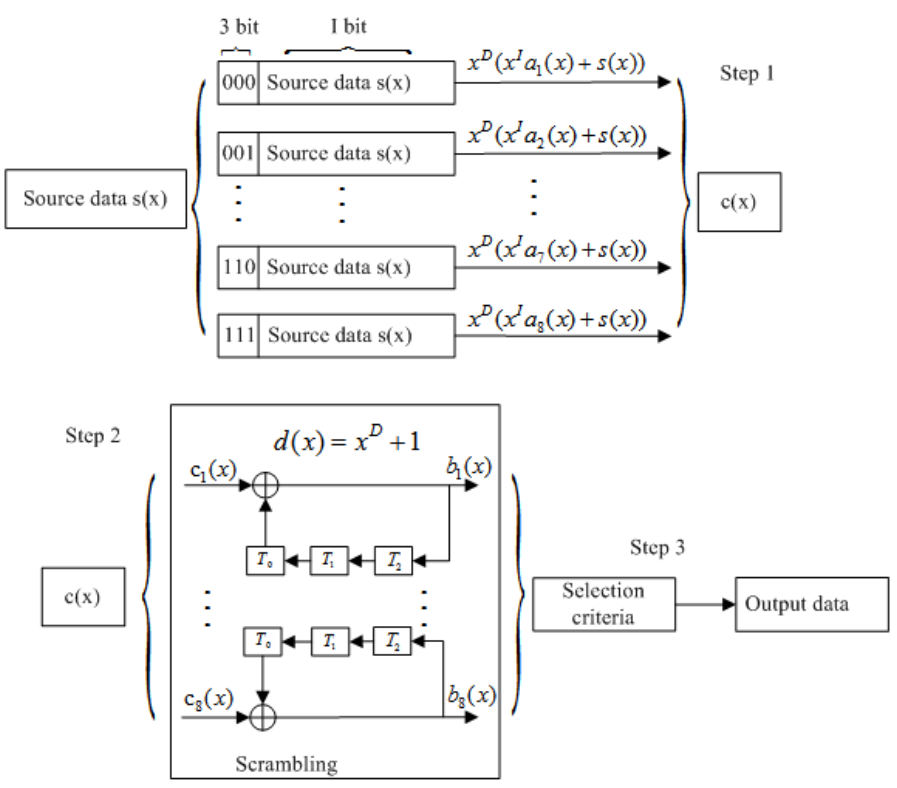

Figure 1.64B/67B coding process

\section{IMPLEMENTATION}

\subsection{Frame}

The hardware implementation is mainly consist of encoder and decoder 2 blocks; specifically encoder can be divided into source data generation, scrambler, and selection \& transition three parts; decoder can be divided into receiver, descrambler, 
and transition three parts. [5] Figure 2 shows the internal structure of encoder and decoder.
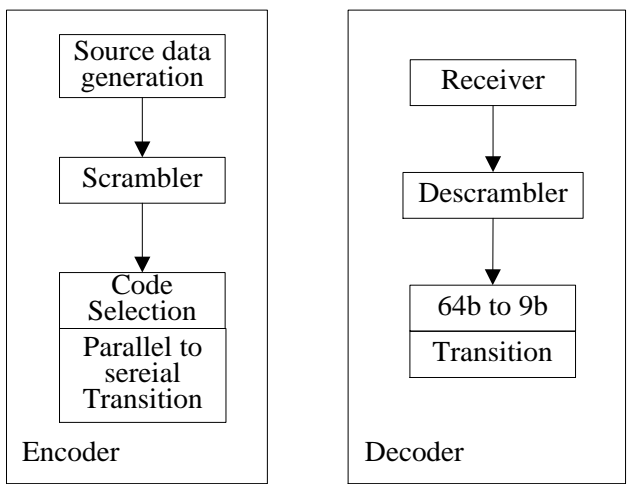

Figure 2. Internal structure of encoder and decoder

\subsection{Encoder}

There should be no clearance between characters in the channel. So encoder needs to send idle characters in the source data generation part when there is no signal data appears.

\subsubsection{Scrambler}

Scrambling was implemented using a series of interconnected delay elements and XOR gates, as shown in Figure 3.

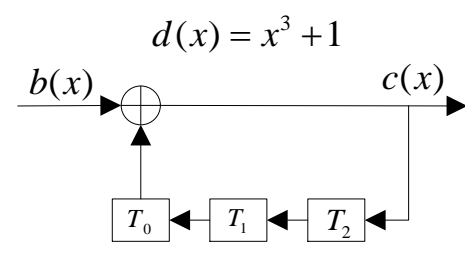

Figure 3. Scrambling process

\subsubsection{State machine for code selection}

Absolute RDS uses absolute operation to replace square operation, its performance is similar to minimum squared weight (MSW).

Figure 4 displays the state machine in FPGA to realize optimal code selection. Its main work focus on obtaining the candidate codes' ABSRDS values and select the optimal code by comparing their ABSRDS values.

\subsection{Decoder}

The most important part of decoder is descrambler, which works to translate the code received into original data. It is the inverse process of scrambling.

\subsubsection{Descrambler}

Similar to scrambler, descrambler was implemented using a series of interconnected delay elements and XOR gates, the descrambling process is shown in Figure 5.

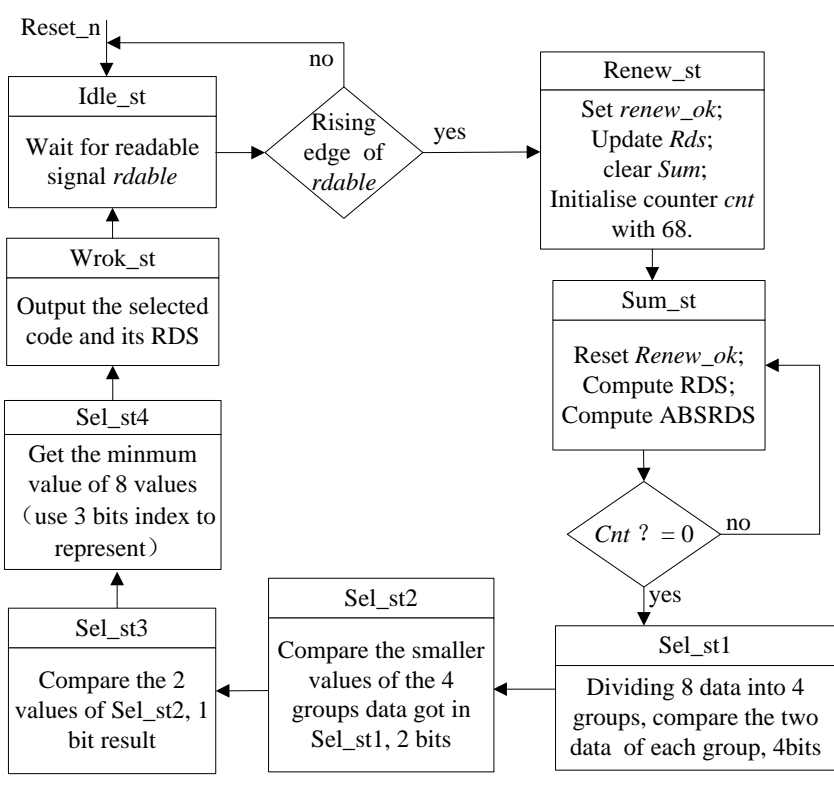

Figure 4. State machine for optical code selection

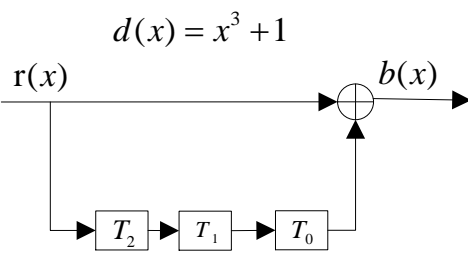

Figure 5. Descrambling process

\subsection{FPGA logic resource occupation}

$8 \mathrm{~B} / 10 \mathrm{~B}$ coding scheme is one of the superior modulation coding schemes, and it has been used in digital cable communication for a long time. In order to assess the performance of $64 \mathrm{~B} / 67 \mathrm{~B}$ coding scheme, we implement both of these two kinds of coding schemes respectively on the same FPGA circuit to make evaluation on their performance.

Table 1 shows the percent of logic resource expense of $8 \mathrm{~B} / 10 \mathrm{~B}$ and $64 \mathrm{~B} / 67 \mathrm{~B}$.

Table 1 Logic resource expense of 8B/10B and 64B/67B

\begin{tabular}{|c|c|c|c|}
\hline & $\begin{array}{c}\text { Encoder logic } \\
\text { resource } \\
\text { expense (LE) }\end{array}$ & $\begin{array}{c}\text { Decoder logic } \\
\text { resource } \\
\text { expense (LE) }\end{array}$ & $\begin{array}{c}\text { Percent } \\
\text { of total } \\
\text { resource }\end{array}$ \\
\hline 8B/10B & 42 & 39 & $0.3 \%$ \\
\hline 64B/67B & $70^{*}$ & 67 & $0.4 \%$ \\
\cline { 3 - 4 } & $1365^{* *}$ & $6.3 \%$ \\
\hline
\end{tabular}

* Scrambler, ** Optimal code selection.

The scrambler in $64 \mathrm{~B} / 67 \mathrm{~B}$ coding scheme just consumed $0.4 \%$ of all system logic resource, is almost the same as $8 \mathrm{~B} / 10 \mathrm{~B}$ coding scheme. But the code selection part consumed $4.3 \%$ of the system logic resource. Since $4.7 \%$ resource occupation is still a small scale for FPGA core, it can meet the design requirements of seismic data acquisition system. 


\section{VERIFICATION}

The developed 64B/67B code had been applied to our large scale seismic data acquisition system. Experiments had been made to test its performance. Figure 6 displays the picture of the experiment system and Figure 7 shows its block diagram.

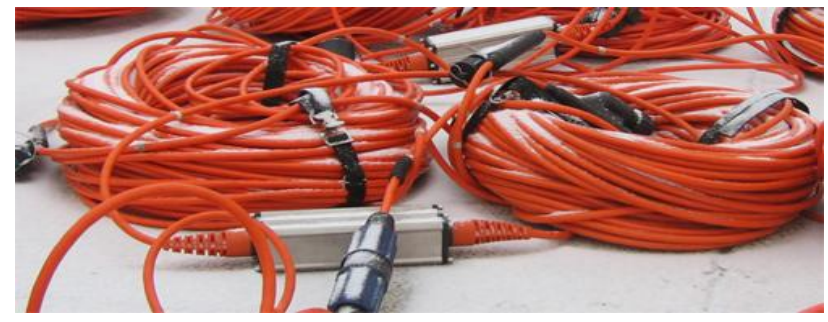

Figure 6. FPGA implement

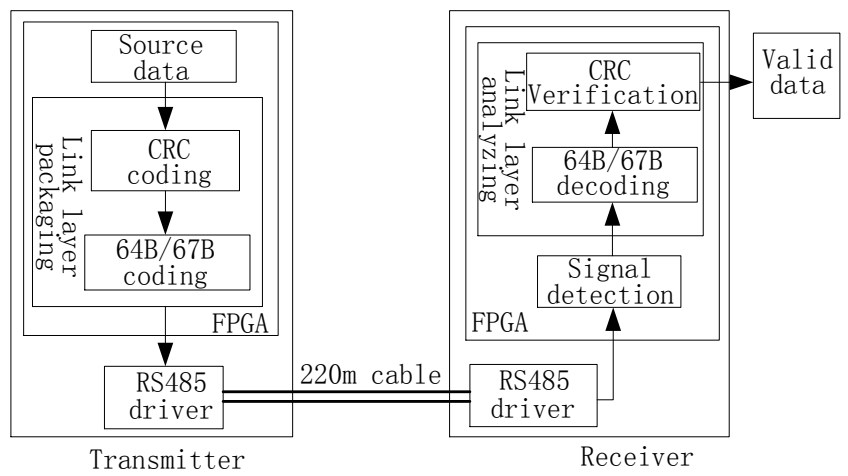

Figure 7. Frame of testing system

\subsection{Bit error rate}

We can easily get the bit error rate by minoring the CRC model. Table 2 shows the testing results at $5 \mathrm{MHz}$ and $8 \mathrm{MHz}$. When the clock frequency is $5 \mathrm{MHz}$, the bit error rate of both $8 \mathrm{~B} / 10 \mathrm{~B}$ and 64B/67B coding scheme are 0 . When clock frequency is $8 \mathrm{MHz}$, the bit error rate is $2.4 \times 10^{-5}$, but it can be eliminated by error correction model in the system.

Table 2. Bit error rate at $5 \mathrm{MHz}$ and $8 \mathrm{MHz}$

\begin{tabular}{|c|c|c|}
\hline & $5 \mathrm{MHz}$ & $8 \mathrm{MHz}$ \\
\hline $8 \mathrm{~B} / 10 \mathrm{~B}$ & $0 \%$ & $0 \%$ \\
\hline $64 \mathrm{~B} / 67 \mathrm{~B}$ & $0 \%$ & $2.4 \times 10^{-5}$ \\
\hline
\end{tabular}

\subsection{Effective transmission speed}

In the effective transmission speed testing process, we set the frame length of source data as 200 bytes. According to the $8 \mathrm{~B} / 10 \mathrm{~B}$ and $64 \mathrm{~B} / 67 \mathrm{~B}$ encoding rules, it is necessary to insert synchronization characters in front of each data frame. So the effective bit rate will be less than $8 / 10$ and 64/67. Taking the synchronization characters into consideration, the effective bit rate of these two coding scheme mentioned above should have the relation below,
$8 \mathrm{~B} / 10 \mathrm{~B}$, effective bit rate, $\frac{200 * 8}{205 * 10}=0.780$;

$64 \mathrm{~B} / 67 \mathrm{~B}$, effective bit rate, $\frac{200 * 8}{26 * 67}=0.918$;

$v_{64 B 67 B} / v_{8 B 10 B}=\frac{0.918}{0.78}=1.178$

Theoretically, the effective transmission speed of $64 \mathrm{~B} / 67 \mathrm{~B}$ should be 1.178 times faster than $8 \mathrm{~B} / 10 \mathrm{~B}$ coding scheme when works at the same clock frequency.

Table 3 presents the effective data transmission speed in testing experiments.

Table 3. Effective data transmission speed at $8 \mathrm{MHz}$

\begin{tabular}{|c|c|}
\hline Frame/Second & $8 \mathrm{MHz}$ \\
\hline$v_{8 B 10 B}$ & 3190 \\
\hline$v_{64 B 67 B}$ & 3682 \\
\hline$v_{64 B 67 B} / v_{8 B 10 B}$ & 1.154 \\
\hline
\end{tabular}

It is easy to find that the effective transmission speed of $64 \mathrm{~B} / 67 \mathrm{~B}$ is 1.15 times faster than $8 \mathrm{~B} / 10 \mathrm{~B}$. The effective transmission speed has been improved by $15 \%$.

The difference between theoretical values (1) with the values measured is caused by the idle characters for channel sequence padding.

\section{CONCLUSIONS}

The hardware implementation based on FPGA presented proves that $64 \mathrm{~B} / 67 \mathrm{~B}$ coding technique is practical in seismic data acquisition system. By comparing the source occupation status, bit error rate and effective transmission speed, we assessed the performance of these 3 kinds of coding scheme. And 64B/67B channel code is highly suitable for seismic data acquisition system.

\section{REFERENCES}

[1] Fair, I.J. \&Grover, W.D. 1991. Guided scrambling: a new line coding technique for high bit rate fiber optic transmission systems. Communications, IEEE Transactions 39(2): 289-297.

[2] Xue, Yang et al. 2012. A high-bit-rate (64/67) DC-balance line code scheme based on guided scrambling. Applied Mechanics and Materials 229-231: 1572-1576.

[3] Fair, I.J. \& Wang, Q. 1995. Polynomials for guided scrambling line codes. Selected Areas in Communications, IEEE Journal 13(3): 499-509.

[4] Immink, S.A. \& Wilson, H.Y. 2000. A comparison of two schemes for generating DC-free RLL sequences. IEEE International Symposium on Information Theory. Sorrento: IEEE.

[5] Hua, X.H. \& Chen, D.L. 2008. The Design and Simulation Based on Scrambler and Descrambler of AHDL. Journal of Huangshi Institute of Technology 2008 (3): 53-56. 